\title{
On Fuzzy Primal Exterior Point Simplex Algorithm for Solving Linear Programming Problems with Fuzzy Numbers
}

\author{
E. M. Badr \\ Scientific Computing Department, Faculty of Computers \& Informatics, Benha University, \\ Benha, Egypt. Email Address: badrgraph@gmail.com
}

\begin{abstract}
Linear programming problems with fuzzy variables have been investigated by many researchers in the recent literature. Some methods to solve these problems such as the primal simplex method and the dual simplex method, are based on the concept of comparison of fuzzy numbers by using ranking functions. In this paper, We first introduce a new method fuzzy exterior point simplex algorithm for solving fuzzy number linear programming problems, by use of linear ranking function. We state our recently established results and develop the fuzzy exterior point simplex algorithm. Finally, we give an illustrative example.
\end{abstract}

Keywords : Fuzzy linear programming, ranking function, exterior point simplex algorithm.

\section{Introduction}

Fuzzy set theory has been applied to many disciplines such as control theory, management sciences, mathematical modelling and industrial applications. The concept of fuzzy mathematical programming in the general level was first proposed by Tanaka et al. [1].The first formulation of fuzzy linear Programming (FLP) was proposed by Zimmermann [2]. Afterwards, many authors considered various types of 
the FLP problems and proposed several approaches for solving these problems .Many convenient methods are based on the concept of comparison of fuzzy numbers using ranking functions $[3,4,5,6,7,8]$. Nasseri et al. [8] introduced a primal simplex algorithm for solving the linear programming problems with trapezoidal fuzzy variables. Mahdavi-Amiri and Nasseri [6] introduced a dual simplex algorithm for solving the linear programming problems with trapezoidal fuzzy variables. Ganesan and Veeramani [9] introduced a new approach for solving a kind of linear programming involving symmetric trapezoidal fuzzy numbers without converting them to the crisp linear programming problems. Nasseri and Mahdavi-Amiri [7] based on this approach defined a new concept of duality of these problems. Ebrahimnejad and Nasseri [10] used the complementary slackness to solve fuzzy linear programming without the need for a simple tableau.

In this paper, We first explain the concept of the comparison of fuzzy numbers by introducing a linear ranking function. Moreover, we describe basic feasible solution for the Fuzzy Number Linear Programming (FNLP) problems and we propose the Exterior Point Simplex Algorithm (EPSA), which was first proposed by Paparrizos [11].

\section{Definitions and Notations}

The aim of this section is to present some notations and results which are of useful in our further consideration.

\section{A. Fuzzy Sets}

Let $X$ be a classical set of objects, called the universe, whose generic elements are denoted by $x$. The membership in a crisp subset of $X$ is often viewed as characteristic function $\mu_{A}(x)$ from $X$ to $\{0,1\}$ such that:

$$
\mu_{A}(x)= \begin{cases}1 & , \text { if } x \in A \\ 0 & , \text { otherwise }\end{cases}
$$

where $\{0,1\}$ is called a valuation set. 
If the valuation set is allowed to be the real interval $[0,1], A$ is called a fuzzy set proposed by Zadeh [11]. $\mu_{A}(x)$ is the degree of membership of $x$ in $A$. The closer the value of $\mu_{A}(x)$ is to 1 , the more $x$ belong to $A$. Therefore, $A$ is completely characterized by the set of ordered pairs: $A=\left\{\left(x, \mu_{A}(x) \mid x \in X\right\}\right.$.

The support of a fuzzy set $A$ is the crisp subset of $X$ and is presented as:

$$
\operatorname{Supp}(A)=\left\{x \in X \mid \mu_{A}(x)>0\right\}
$$

The $\alpha$-level ( $(\alpha-c u t)$ set of a fuzzy set $A$ is a crisp subset of $X$ and is denoted by

$$
A_{\alpha}=\left\{x \in X \mid \mu_{A}(x) \geq \alpha\right\}
$$

A fuzzy set $A$ in $X$ is convex if :

$$
\mu_{A}(\lambda x+(1-\lambda) y) \geq \min \left\{\mu_{A}(x), \mu_{A}(y)\right\} \text { such that } x, y \in X \text { and }[0,1] \in \lambda .
$$

Alternatively, a fuzzy set is convex if all $\alpha$-level sets are convex. Note that in this paper we suppose that $X=R$.

A fuzzy number $A$ is a convex normalized fuzzy set on the real line $R$ such that :

1- It exists at least one $x_{0} \in R$ with $\mu_{A}(x)=1$.

2- $\mu_{A}(x)$ is piecewise continuous.

Among the various types of fuzzy numbers, triangular and trapezoidal fuzzy numbers are of the most important. Note that, in this study we only consider trapezoidal fuzzy numbers. A fuzzy number is a trapezoidal fuzzy number if the membership function of it be in the following form:

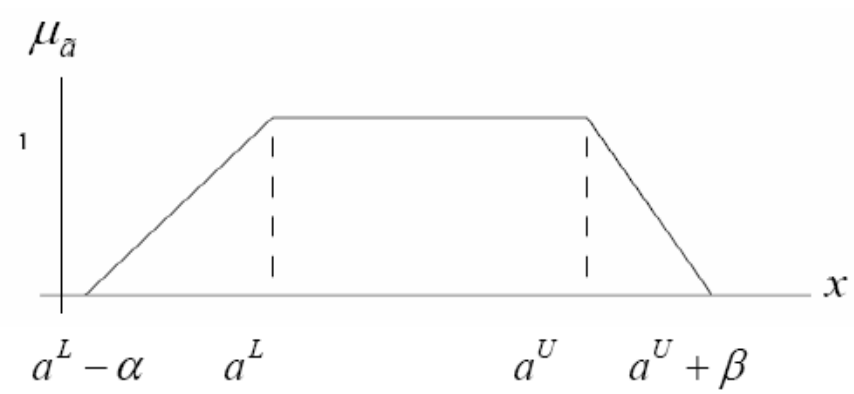

Fig. 1 Trapezoidal Fuzzy Number

We show any trapezoidal fuzzy number by, $a=\left(a^{L}, a^{U}, \alpha, \beta\right)$ where the support of $a$ is $\left(a^{L}-\alpha, a^{U}+\beta\right)$, and the modal set of $a$ is $\left[a^{L}, a^{U}\right]$. Let $F(R)$ be the set of trapezoidal fuzzy numbers. In the next subsection we describe arithmetic on $F(R)$.

\section{Arithmetic on Fuzzy Numbers ( on trapezoidal fuzzy numbers )}


Let $a=\left(a^{L}, a^{U}, \alpha, \beta\right)$ and $\tilde{b}=\left(b^{L}, b^{U}, \gamma, \theta\right)$ be two trapezoidal fuzzy numbers and $x \in R$. Then, the results of applying fuzzy arithmetic on the trapezoidal fuzzy numbers as shown in the following:

(i) Image of $\tilde{a}:-\tilde{a}=\left(-a^{U},-a^{L}, \beta, \alpha\right)$

(ii) Addition: $\tilde{a}+\tilde{b}=\left(a^{L}, a^{U}, \alpha, \beta\right)+\left(b^{L}, b^{U}, \gamma, \theta\right)$

$$
=\left[a^{L}+b^{L}, a^{U}+b^{U}, \alpha+\gamma, \beta+\theta\right]
$$

(iii) Subtraction: $\tilde{a}-\tilde{b}=\tilde{a}+(-\tilde{b})=\left(a^{L}, a^{U}, \alpha, \beta\right)+\left(-b^{U},-b^{L}, \theta, \gamma\right)$

$$
=\left(a^{L}-b^{U}, a^{U}-b^{L}, \alpha+\theta, \beta+\gamma\right)
$$

(iv) Scalar Multiplication:

$$
\begin{aligned}
& x>0, \quad x \tilde{a}=\left[x a^{L}, x a^{U}, x \alpha, x \beta\right] \\
& x<0, \quad x \tilde{a}=\left[x a^{U}, x a^{L},-x \beta,-x \alpha\right]
\end{aligned}
$$

\section{4- Ranking Functions}

Ranking is a viable approach for ordering fuzzy numbers. Various types of ranking functions have been introduced and some have been used for solving linear programming problems with fuzzy parameters $[3,4,6,7,12]$. A review of some common methods for ranking fuzzy subsets of unit interval can be seen in [13].

Here, we deal with ranking the elements of $F(R)$. In fact, an effective approach for ordering the elements of $F(R)$ is to define a ranking function $R: F(R) \rightarrow(R)$ mapping trapezoidal fuzzy numbers into $R$. Consider $\tilde{a}$ and $\tilde{b}$ in $F(R)$. Define order on $F(R)$ as follows $[6,7]$ :

$$
\begin{aligned}
& \tilde{a} \underset{\Re}{\mathfrak{b}} \quad \text { if } \mathfrak{R}(\tilde{a}) \geq \mathfrak{R}(\tilde{b}) \text {, } \\
& \tilde{a}>\tilde{b} \quad \text { if } \quad \mathfrak{R}(\tilde{a})>\mathfrak{R}(\tilde{b}),
\end{aligned}
$$




$$
\tilde{a}=\tilde{b} \quad \text { if } \mathfrak{R}(\tilde{a})=\mathfrak{R}(\tilde{b}),
$$

Where $\tilde{a}$ and $\tilde{b}$ are in $F(R)$. Also we write $\tilde{a} \leq \tilde{b}$ if $\tilde{b} \geq \tilde{a}$. Then, for any linear

ranking $\Re$ we may obtain: $\tilde{a} \underset{\Re}{\geq}$ if and only if $\tilde{a}-\tilde{b} \underset{\Re}{\geq} \tilde{0}$,or if and only if $-\tilde{b} \underset{\Re}{\gtrless}-\tilde{a}$.

Also, if $\tilde{a} \underset{\Re}{\geq}$ and $\tilde{c} \underset{\Re}{\geq}$, then $\tilde{a}+\tilde{c} \underset{\Re}{\geq} \tilde{b}+\tilde{d}$.

One suggestion for a linear ranking function as following:

$$
\mathfrak{R}(\tilde{a})=a^{L}+a^{U}+\frac{1}{2}(\beta-\alpha)
$$

where $\tilde{a}=\left[a^{L}, a^{U}, \alpha, \beta\right] \in F(R)$.

\section{5- FUZZY LINEAR PROGRAMMING}

In this section, we introduce fuzzy linear programming (FLP) problems. So, we first define linear programming problems.

\section{A. Linear Programming}

A linear programming (LP) problem is defined as:

$$
\begin{array}{ll}
\operatorname{Max} & z=c x \\
\text { s.t. } & A x=b \\
& x \geq 0
\end{array}
$$

where $c=\left(c_{1}, c_{2}, \ldots, c_{n}\right), b=\left(b_{1}, b_{2}, \ldots, b_{n}\right)^{T}$ and $A=\left[a_{i j}\right]_{m \times n}$.

In the above problem, all of the parameters are crisp [14]. Now, if some of the parameters be fuzzy numbers we obtain a fuzzy linear programming which is defined in the next subsection.

\section{B. Fuzzy Linear Programming}

Suppose that in the linear programming problem some parameters be fuzzy numbers. Hence, it is possible that some coefficients of the problem in the objective function, technical coefficients, the right-hand side coefficients or decision making variables be fuzzy number $[4,5,15,16,17,18,19]$. Here, we consider the linear programming problems with fuzzy numbers in the objective function. 


\section{6- FUZZY NUMBER LINEAR PROGRAMMING}

A fuzzy number linear programming (FNLP) problem is defined as follows:

$$
\begin{array}{ll}
\operatorname{Max} & z \underset{\Re}{=} \tilde{c} x \\
\text { s.t. } & A x=b \\
& x \geq 0
\end{array}
$$

Where $b \in R^{m}, x \in R^{n}, A \in R^{m \times n}, \tilde{c}^{T} \in(F(R))^{n}$ and $\mathfrak{R}$ is a linear ranking function.

Now, we describe a fuzzy primal exterior point simplex algorithm ( FPEPSA), which solve FNLP problem directly on the primal simplex tableau.

The algorithm begins with a basic feasible point . we find the set of non-basic indices $J_{-}=\left\{j: a_{0 j}<0\right\}$. If $\mathbf{J}_{-}=\varnothing$, then the first main point is most optimal and the algorithm stops. Otherwise, we calculate $a_{00}=\sum_{j \in J_{-}} a_{i j}$ and $a_{i 0}=\sum_{j \in J_{-}} a_{i j}$ where $i=$ $1,2, \ldots, m$ after that find the set of indices $I_{+}=\left\{i: a_{i 0}>0\right\}$. If $I_{+}=\varnothing$, then the problem is unbounded. Otherwise, we determine the index of entering variable $r$ from ratio test $\frac{b_{r}}{a_{r} 0}=\min \left\{\frac{b_{i}}{a_{i} 0}: i \in I_{+}\right\}$. In order to find the index of the leaving variable $s$, we must find the set of indices $J_{+}=\left\{j: a_{0 j}>0\right\}$ and after that we calculate $\theta_{1}$ and $\theta_{2}$. If $\theta_{1} \leq \theta_{2}$ put $s=k$ otherwise $s=1$. he last step is the pivoting by the pivot element $a_{r s}$.

EPSA constructs two paths to the optimal solution. One path consists of basic but not feasible solutions; so this is an "exterior path". The second path is feasible. It consists of line segments, the endpoints of which lie on the boundary of the feasible region. 


\section{Exterior Point Simplex Algorithm Description}

\section{tep0: (Initialization)}

Start with a feasible basic point and construct the corresponding tableau exterior simplex.

\section{Step1: (Test of termination)}

Find the set $J_{-}=\left\{j: a_{0 j}<0\right\}$. If $\mathbf{J}_{-}=\varnothing$,STOP. The problem is optimal.

Otherwise, calculate $a_{00}=\sum_{j \in J_{-}} a_{i j}$ and $a_{i 0}=\sum_{j \in J_{-}} a_{i j}$ where $i=1,2, \ldots, m$

\section{Step2: (Choice of entering variable)}

Find the set $\mathrm{I}_{+}=\left\{\mathrm{i}: \mathrm{a}_{\mathrm{i} 0}>0\right\}$. If $\mathrm{I}_{+}=\varnothing, \mathrm{STOP}$. The problem is unbounded.

Otherwise, determine the index of entering variable $r$ from the relation

$$
\frac{b_{r}}{a_{r 0}}=\min \left\{\frac{b_{i}}{a_{i}}: i \in I_{+}\right\}
$$

\section{Step3: (Choice of leaving variable)}

Put $\quad J_{+}=\left\{j: a_{0 j}>0\right\}$ and calculate

$$
\begin{aligned}
& \theta_{1}=\frac{-a_{0 k}}{a_{r k}}=\min \left\{\frac{-a_{0 j}}{a_{r j}}: j \in J_{-}, a_{r j}>0\right\} \\
& \theta_{2}=\frac{-a_{0 l}}{a_{r l}}=\min \left\{\frac{-a_{0 j}}{a_{r j}}: j \in J_{+}, a_{r j}<0\right\}
\end{aligned}
$$

Find the index of the leaving variable $s$, if $\theta_{1} \leq \theta_{2}$ put $\mathrm{s}=\mathrm{k}$ otherwise $\mathrm{s}=1$.

\section{Step4: (Pivoting)}

Form the next tableau by the pivoting variable $a_{r s}$ i.e.

$$
\text { Put } \quad a_{r j} \leftarrow \frac{a_{r j}}{a_{r s}} \quad \text { where } j=1,2, \ldots, n, n+1
$$

$a_{i j} \leftarrow a_{i j}-\frac{a_{r j}}{a_{r s}} a_{i s}$ where $i=0,1,2, \ldots m(i \neq r)$ and $j=1,2, \ldots, n, n+1$

If $\mathrm{s}=\mathrm{k}$ put $\mathrm{a}_{0 \mathrm{r}} \leftarrow \mathrm{a}_{0 \mathrm{r}}-1$.

and go to Step1. 


\section{A Numerical Example}

For an illustration of the above method we solve FNLP problem by use of Fuzzy Primal Exterior Point Simplex Algorithm ( FPEPSA ).

\section{Example 7.1.}

$$
\begin{array}{ll}
\max \tilde{z} \underset{\Re}{ }=(2,4,2,6) x_{1}+(2,6,1,3) x_{2}+(1,3,1,3) x_{3} \\
\text { s.t. } \quad x_{1}+x_{2}+2 x_{3}+x_{4}=2 \\
& 2 x_{1}+3 x_{2}+4 x_{3}+x_{5}=3 \\
& 6 x_{1}+6 x_{2}+2 x_{3}+x_{6}=8 \quad \text { where : } x_{\mathrm{i}} \geq 0, \mathrm{i}=1,2,3,4,5,6
\end{array}
$$

Step (0): we construct the initial tableau of exterior simplex:

\begin{tabular}{|c|c|ccc|ccc|c|}
\hline Basis & & $x_{1}$ & $x_{2}$ & $x_{3}$ & $x_{4}$ & $x_{5}$ & $x_{6}$ & R.H.S \\
\hline $\mathrm{Z}$ & $(-13,-5,12,4)$ & $(-4,-2,6,2)$ & $(-6,-2,3,1)$ & $(-3,-1,3,1)$ & 0 & 0 & 0 & 0 \\
\hline$x_{4}$ & 4 & 1 & 1 & 2 & 1 & 0 & 0 & 2 \\
\hline$x_{5}$ & 9 & 2 & 3 & 4 & 0 & 1 & 0 & 3 \\
\hline$x_{6}$ & 14 & 6 & 6 & 2 & 0 & 0 & 1 & 8 \\
\hline
\end{tabular}

$\left(\tilde{z_{1}}-\mathrm{c}_{1}, \tilde{z_{2}}-\mathrm{c}_{2}, \tilde{z_{3}}-\mathrm{c}_{3}\right) \underset{R}{=}((-2,-4,6,2),(-4,-4,3,1),(-2,-2,3,1))$ and $\left(\gamma_{1}, \gamma_{2}, \gamma_{3}\right)=\left(\mathfrak{R}\left(\gamma_{1}\right), \mathfrak{R}\left(\gamma_{2}\right), \mathfrak{R}\left(\gamma_{3}\right)\right)=(-8,-9,-5)$.

Step (1):

$$
J-=\left\{j: \mathrm{a}_{\mathrm{oj}} \underset{R}{<} 0\right\}=\{1,2,3\} \neq 1 \text { the Algorithm does not stop. }
$$

Step (2): $I_{+}=\left\{i: a_{\text {io }}>0\right\}=\{1,2,3\} \neq \Phi$ the problem is not unbounded

$$
\frac{b r}{a_{r o}}=\min \left\{\frac{b i}{\text { aio }}, i \in I_{+}\right\}=\min \left\{\frac{b_{1}}{a_{10}}, \frac{b_{2}}{a_{20}}, \frac{b 3}{a_{30}}\right\}=\min \left\{\frac{1}{2}, \frac{1}{3}, \frac{4}{7}\right\}=\frac{1}{3} \Rightarrow r=2
$$

Step (3): $\mathrm{J}_{+}=\left\{\mathrm{j}: \mathrm{a}_{\mathrm{oj}} \underset{R}{>} 0\right\}=\Phi$

$$
\begin{aligned}
& \theta_{1}=\frac{-a_{o k}}{a_{r k}} \underset{R}{=} \min \left\{\frac{-a o j}{a r j}=j \in J-, a r j>0\right\}=\min \left\{\frac{-a o l}{a_{21}}, \frac{-a o 2}{a_{22}}, \frac{-a o 3}{a_{23}}\right\}=\min \{(1,2,1,3), \\
& \left.\left(\frac{2}{3}, 2, \frac{1}{3}, 1\right),\left(\frac{1}{4}, \frac{3}{4}, \frac{1}{4}, \frac{3}{4}\right)\right\}
\end{aligned}
$$




$$
\begin{aligned}
& R\left(\theta_{1}\right)=\min \left\{R(2,1,1,3), R\left(\frac{4}{3}, \frac{4}{3}, \frac{1}{3}, 1\right), R\left(\frac{1}{2}, \frac{1}{2}, \frac{1}{4}, \frac{3}{4}\right)\right\}=\min \left\{4,3, \frac{5}{4}\right\}=\frac{5}{4} \\
& \therefore \mathrm{k}=3 \\
& \theta_{2}=\frac{-a o L}{\operatorname{arL}}=\min \left\{\frac{-a_{o j}}{\operatorname{arj}}: j \in J_{+}, \operatorname{arj}<o\right\} \Rightarrow R\left(\theta_{2}\right)=\min \{\Phi\}=\infty \\
& \Rightarrow R\left(\theta_{1}\right)<R\left(\theta_{2}\right) \Rightarrow \theta_{1}<_{R} \theta_{2} \Rightarrow \mathrm{s}=\mathrm{k}=3
\end{aligned}
$$

the pivot element is $\mathrm{a}_{23}$

Step (4): the next tableau by pivot element:

\begin{tabular}{|c|c|c|c|c|c|c|c|c|}
\hline Basis & $x_{1}$ & $x_{2}$ & $x_{3}$ & $x_{4}$ & $x_{5}$ & $x_{6}$ & R.H.S \\
\hline $\mathrm{Z}$ & $\left(-5,-3, \frac{42}{4}, \frac{30}{4}\right)$ & $\left(-3,-1, \frac{13}{2}, \frac{7}{2}\right)$ & $\left(-2,-3, \frac{15}{4}, \frac{13}{4}\right)$ & $\tilde{0}$ & 0 & $\left(0,1, \frac{1}{4}, \frac{3}{4}\right)$ & 0 & $\left(0,3, \frac{3}{4}, \frac{9}{4}\right)$ \\
\hline$x_{4}$ & $\frac{-1}{2}$ & 0 & $\frac{-1}{2}$ & 0 & 1 & $\frac{-1}{2}$ & 0 & $\frac{1}{2}$ \\
\hline$x_{3}$ & $\frac{5}{4}$ & $\frac{1}{2}$ & $\frac{3}{4}$ & 1 & 0 & $\frac{1}{4}$ & 0 & $\frac{3}{4}$ \\
\hline$x_{6}$ & $\frac{19}{2}$ & 5 & $\frac{9}{2}$ & 0 & 0 & $\frac{-1}{2}$ & 1 & $\frac{13}{2}$ \\
\hline
\end{tabular}

Step (1): $\mathrm{J}_{-}=\left\{\mathrm{j}: \mathrm{a}_{\mathrm{oj}} \underset{R}{<} 0\right\}=\{1,2\} \Rightarrow$ the Algorithm does not stop.

$$
\left(\gamma_{1}, \gamma_{2}, \gamma_{5}\right)=\left(R\left(\gamma_{1}\right), R\left(\gamma_{2}\right), R\left(\gamma_{5}\right)\right)=\left(\frac{-11}{2}, \frac{-21}{4}, \frac{5}{4}\right)
$$

Step (2): $\mathrm{I}_{+}=\left\{\mathrm{i}: \mathrm{a}_{\mathrm{io}}>0\right\}=\{2,3\} \neq \Phi \Rightarrow$ the problem is bounded

$$
\frac{b r}{a_{r o}}=\min \left\{\frac{b i}{a_{i o}}, i \in I_{+}\right\}=\min \left\{\frac{b_{2}}{a_{20}}, \frac{b_{3}}{a_{30}}\right\}=\min \left\{\frac{3}{5}, \frac{13}{19}\right\}=\frac{3}{5} \Rightarrow r=2<
$$

Step (3): $\mathrm{J}_{+}=\left\{\mathrm{j}: \mathrm{a}_{\mathrm{oj}} \underset{R}{>} 0\right\}=\{5\}$

$$
\theta_{1} \stackrel{\bar{R}}{\frac{-a_{o k}}{a_{r k}}}=\min \left\{\frac{-a o j}{a r j}: j \in J, a r_{j}>0\right\}=\min \left\{\frac{-a o l}{a_{21}}, \frac{-a o 2}{a_{22}}\right\}=\min \left\{(6,2,7,13\},\left(\frac{10}{3}, \frac{10}{3}, \frac{13}{3},\right)\right.
$$




$$
\begin{aligned}
& R\left(\theta_{1}\right)=\min \left\{R(6,2,7,13), R\left(\frac{10}{3}, \frac{10}{3}, \frac{13}{3}, 5\right)\right\}=\min \{11,7\}=7 \Rightarrow k=2 \\
& \theta_{2}=\frac{-a_{o k}}{a_{r L}}=\min \left\{\frac{-a o j}{a r j}: j \in J, a r j<0\right\} \Rightarrow R\left(\theta_{2}\right)=\min \{\Phi=\infty\} \\
& \Rightarrow R\left(\theta_{1}\right)<R\left(\theta_{2}\right) \Rightarrow \theta_{1} \underset{R}{<} \theta_{2} \Rightarrow \mathrm{s}=\mathrm{k}=2
\end{aligned}
$$

the pivot element is $a_{22}$

Step (4): the next tableau by pivot element

\begin{tabular}{|c|c|c|c|c|c|c|c|c|}
\hline Basis & $x_{1}$ & $x_{2}$ & $x_{3}$ & $x_{4}$ & $x_{5}$ & $x_{6}$ & R.H.S \\
\hline $\mathrm{Z}$ & $\left(\frac{-4}{3}, \frac{2}{3}, \frac{26}{3}, 6\right)$ & $\left(\frac{-4}{3}, \frac{2}{3}, \frac{26}{3}, 6\right)$ & $\tilde{0}$ & $\left(\frac{8}{3}, 4, \frac{13}{3}, 5\right)$ & 0 & $\left(\frac{2}{3}, 2, \frac{4}{3}, 2\right)$ & 0 & $(3,5,4,6)$ \\
\hline$x_{4}$ & $\frac{1}{3}$ & $\frac{1}{3}$ & 0 & $\frac{2}{3}$ & 1 & $\frac{-1}{3}$ & 0 & 1 \\
\hline$x_{2}$ & $\frac{2}{3}$ & $\frac{2}{3}$ & 1 & $\frac{4}{3}$ & 0 & $\frac{1}{3}$ & 0 & 1 \\
\hline$x_{6}$ & 2 & 2 & 0 & -6 & 0 & -2 & 1 & 2 \\
\hline
\end{tabular}

Step (1): $\mathrm{J}_{-}=\left\{\mathrm{j}: \mathrm{a}_{\mathrm{oj}}{ }_{R}^{<0}\right\}=\{1\} \neq \Phi \Rightarrow$ the algorithm does not stop .

$$
\left(\gamma_{1}, \gamma_{3}, \gamma_{5}\right)=\left(R\left(\gamma_{1}\right), R\left(\gamma_{3}\right), R\left(\gamma_{5}\right)\right)=(-2,7,3)
$$

Step (2): $\mathrm{I}_{+}=\left\{\mathrm{i}: \mathrm{a}_{\mathrm{io}}>0\right\}=\{1,2,3\} \neq \Phi \Rightarrow$ the problem is bounded Ratio test: $\frac{b r}{a_{r o}}=\min \left\{\frac{b i}{a_{i o}}, i \in I_{+}\right\}=\min \left\{\frac{b_{1}}{a_{10}}, \frac{b 2}{a_{20}}, \frac{b_{3}}{a_{30}}\right\}=\min \left\{3, \frac{3}{2}, 1\right\}=1$

The index of the entering variable is $r=3$

Step (3): $\mathrm{J}_{+}=\left\{\mathrm{j}: \mathrm{a}_{\mathrm{oj}}>_{R} 0\right\}=\{3,5\}$

$$
\begin{aligned}
& \theta_{1}=\frac{-a o k}{a r k} \underset{R}{=} \min \left\{\frac{-a o j}{a r j}: j \in J, a r j>0\right\}=\min \left\{\frac{-a o l}{a_{31}}\right\}=\left(\frac{2}{3}, \frac{-1}{3}, 3, \frac{13}{3}\right) \\
& R\left(\theta_{1}\right)=R\left(\frac{2}{3}, \frac{-1}{3}, 3, \frac{13}{3}\right)=1 \\
& \theta_{2}=\frac{-a o l}{a r L} \underset{R}{=} \min \left\{\frac{-a o j}{a r j}: j \in J, a r j<0\right\}=\min \left\{\left(\frac{5}{9}, \frac{5}{9}, \frac{13}{18}, \frac{5}{6}\right),\left(\frac{2}{3}, \frac{2}{3}, \frac{2}{3}, 1\right)\right\}
\end{aligned}
$$




$$
\begin{aligned}
& R\left(\theta_{2}\right)=\min \left\{R\left(\frac{5}{9}, \frac{5}{9}, \frac{13}{18}, \frac{5}{6}\right), R\left(\frac{2}{3}, \frac{2}{3}, \frac{2}{3}, 1\right)\right\}=\min \left\{\frac{7}{6}, \frac{3}{2}\right\}=\frac{7}{6} \\
& R\left(\theta_{1}\right)<R\left(\theta_{2}\right) \Rightarrow s=k=1
\end{aligned}
$$

the pivot element is $a_{31}$

Step (4): the next tableau by pivot element

\begin{tabular}{|c|c|c|c|c|c|c|c|}
\hline Basis & $x_{1}$ & $x_{2}$ & $x_{3}$ & $x_{4}$ & $x_{5}$ & $x_{6}$ & R.H.S \\
\hline $\mathrm{Z}$ & $\tilde{0}$ & 0 & $\left(0, \frac{1}{2}, 1,2\right)$ & 0 & $\left(0, \frac{1}{2}, 1,2\right)$ & 0 & $(4,6,6,8)$ \\
\hline$x_{4}$ & 0 & 0 & $\frac{5}{3}$ & 1 & 0 & $\frac{-1}{6}$ & $\frac{2}{3}$ \\
\hline$x_{2}$ & 0 & $\frac{1}{3}$ & $\frac{10}{3}$ & 0 & 1 & $\frac{-1}{3}$ & $\frac{1}{3}$ \\
\hline$x_{1}$ & 1 & 0 & -3 & 0 & -1 & $\frac{1}{2}$ & 1 \\
\hline
\end{tabular}

$$
\left(\gamma_{3}, \gamma_{5}\right)=\left(R\left(\gamma_{3}\right), R\left(\gamma_{5}\right)\right)=(1,1)
$$

Step (1): $\mathrm{J}_{-}:\left\{j: a_{o j} \underset{R}{<0}\right\}=\Phi \Rightarrow$ the Algorithm stops .

the solution is $\mathrm{z}=\mathrm{R}(\mathrm{z})=\mathrm{R}\left(\frac{16}{3}, \frac{10}{3}, 10, \frac{44}{3}\right)=11, x_{1}=1, \quad x_{2}=\frac{1}{3}, x_{3}=0, x_{4}=\frac{2}{3}$, $x_{5}=x_{6}=0$

\section{Conclusion}

We considered fuzzy number linear programming problems and introduced a new method fuzzy primal exterior point simplex algorithm (FPEPSA) for solving fuzzy number linear programming problems, by use of linear ranking function. Finally, we gave an illustrative example.

\section{References:}

[1] H. Tanaka, T. Okuda and K. Asai " On Fuzzy Mathematical programming" The Journal of Cybernetics, 3 ( 1974), 37-46. 
[2] H. J. Zimmermann " Fuzzy programming and linear programming with several objective functions, Fuzzy Sets and Systems, 1, 45-55.

[3] L. Campos, J. L. Verdegay " Linear Programming Problems and ranking of Fuzzy sets and Systems", Vol.32 (1989), pp. 1-11.

[4] H. R. Maleki, M. Tata and M. Mashinchi " Linear Programming with Fuzzy Variables" Fuzzy Sets and Systems, Vol. 109 (2000), pp.21-33.

[5] H. R. Maleki " Ranking functions and their applications to fuzzy linear programming" Far East J. Math. Sci., Vol. 4 (2002), pp.283-301.

[6] N. Mahdavi-Amiri and S. H. Nasseri " Duality results and a dual simplex method for linear programming problems with trapezoidal fuzzy variables" Fuzzy sets and systems, Vol. 158 (2007), pp. 1961-1978.

[7] N. Mahdavi-Amiri and S.H. Nasseri " Duality in fuzzy number linear programming by use of a certain linear ranking function " Applied Mathematics and computation, Vol. 180 (2006), pp. 206-216.

[8] S. H. Nasseri, E. Ardil, A. Yazdani and R. Zaefarian " Simplex Methods for solving Linear Programming Problems with Fuzzy Numbers" Transactions on Engineering, Computing and Technology Vol. 10 ( 2005), pp. 284-288.

[9] K. Ganesan and P. Veeramani " Fuzzy linear programming with trapezoidal fuzzy numbers " Ann. Oper. Res., Vol. 13 (2006), pp. 305-315.

[10] Ebrahimnejad

[11] R.E. Bellman and L.A. Zadeh, "Decision making in a fuzzy environment", Management Sci. 17 (1970) 141--164.

[12] Y. J. Lai and C. L. Hwang ( 1992), Fuzzy Mathematical Programming Methods and Applications, Springer, Berlin.

[13] X. Wang and E.Kerre " Reasonable Properties for the Ordering of Fuzzy Quantities " (2 parts), Fuzzy Sets and Systems, (2001) ,118, 375-405.

[14] M.S. Bazaraa, J.J. Jarvis and H.D. Sherali, Linear Programming and Network Flows, John Wiley, New York, Second Edition, 1990.

[15] M. Delgado, J.L. Verdegay, and M.A. Vila, “A general model for fuzzy linear programming”, Fuzzy Sets and Systems 29 (1989) 21--29.

[16] S.C. Fang and C.F. Hu, "Linear programming with fuzzy coefficients in constraint”, Comput. Math. Appl. 37 (1999) 63--76.

[17] N. Mahdavi-Amiri and S.H. Nasseri, "Duality in fuzzy variable linear Programming ", 4th World Enformatika Conference, WEC'05, June 24- 
26, 2005, Istanbul, Turkey.

[18] H. Rommelfanger, R. Hanuscheck and J. Wolf, "Linear programming with fuzzy objective”, Fuzzy Sets and Systems 29 (1989) 31--48.

[19] J.L. Verdegay, "A dual approach to solve the fuzzy linear programming problem”, Fuzzy Sets and Systems 14 (1984) 131--141.

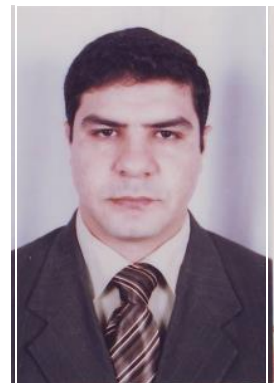

El-Sayed Badr received the B.Sc. (Mathematics) the M.Sc. (Pure Mathematics) degree from Benha University, Benha, Egypt in 1994, 2000 respectively. Also, he received Ph.D. ( Computer Science) degree from Department of Applied Informatics, Macedonia University, Thessaloniki, Greece, in 2006. He is a University associate Professor of Computer Science with the Benha University, Faculty of Computer Science, Department of Scientific Computing. His current research is Parallel Programming, linear programming ,Fuzzy Theory and Graph Theory. 